\title{
Propriedades Psicométricas da Escala de Demandas Percebidas do Trabalho e da Família
}

\author{
Larissa Maria David Gabardo-Martins ${ }^{1}$, Maria Cristina Ferreira \\ Universidade Salgado de Oliveira, Niterói-RJ, Brasil
}

RESUMO

As demandas de trabalho e de família dizem respeito à percepção das responsabilidades do indivíduo com seu trabalho e sua família, respectivamente. Foi objetivo do presente trabalho obter evidências iniciais de da Escala de Demandas Percebidas do Trabalho e da Família. Compuseram a amostra 507 trabalhadores, de ambos os sexos (feminino=56,8\%), com idades variando de 16 a 66 anos $(M=34,26 ; D P=10,02)$. As análises fatoriais confirmatórias revelaram que o modelo de dois fatores correlacionados apresentou os melhores índices de ajuste; e as análises fatoriais confirmatórias multigrupos indicaram que os parâmetros dos itens eram invariantes entre os diferentes grupos analisados. As demandas de trabalho correlacionaram-se positivamente com a sobrecarga no trabalho e as demandas da família, negativamente, com a ambiguidade de papéis na família. As propriedades psicométricas da escala recomendam seu uso futuro em investigações brasileiras destinadas a avaliar as demandas do trabalho e da família.

Palavras-chave: trabalho; família; validade do teste; psicometria.

\section{ABSTRACT - Psychometric Properties of Perceived Work and Family Demand Scale}

The demands of work and family are related to the perception of the responsibilities of individuals with their work and family. This study aimed to obtain initial evidence of validity for the Perceived Demands of Work and Family Scale. The sample consisted of 507 workers, of both sexes (female $=56.8 \%$ ), with ages varying from 16 to 66 years $(M=34.26, S D=10.02)$. Confirmatory factor analyses revealed that the correlated two-factor model presented the best fit indexes; and multi-group confirmatory factor analyses indicated that the parameters of the items were invariant among the different groups analyzed. The work demands correlated positively with work overload, and family demands negatively with the ambiguity of roles in the family. The psychometric properties of the scale suggest its future use in Brazilian investigations aimed at evaluating the demands of work and family.

Keywords: work; family; validity of the test; psychometry.

\section{RESUMEN - Propiedades Psicométricas de La Escala de Demandas Percebidas del Trabajo y de la Familia}

Las demandas de trabajo y de familia se refieren respectivamente a la percepción de las responsabilidades del individuo con su trabajo y con su familia. El objetivo del presente trabajo fue obtener evidencias iniciales de validez de la Escala de Demandas Percibidas del Trabajo y de la Familia. La muestra fue compuesta por 507 trabajadores, de ambos sexos (femenino $=56,8 \%$ ), con edades variando de 16 a 66 años $(M=34,26, S E=10,02)$. Los análisis factoriales confirmatorios revelaron que el modelo de dos factores correlacionados presentó los mejores índices de adaptación; y los análisis factoriales confirmatorios multigrupos indicaron que los parámetros de los ítems eran invariantes entre los diferentes grupos analizados. Las demandas de trabajo se correlacionaron positivamente con la sobrecarga en el trabajo y las demandas de familia, negativamente con la ambigüedad de roles en la familia. Las propiedades psicométricas de la escala recomiendan su futuro uso en investigaciones brasileñas destinadas a evaluar las demandas del trabajo y de la familia.

Palabras clave: trabajo; familia; validez de la prueba; psicometría.

As mudanças sociais e econômicas que ocorreram nas últimas décadas (por exemplo, a inserção da mulher no mercado de trabalho) fizeram com que os dois domínios centrais na vida do indivíduo adulto, quais sejam o trabalho e a família, ficassem intimamente associados (Allen, Cho \& Meier, 2014). Esse fato levou os pesquisadores a se dedicarem mais profundamente ao estudo da interface trabalho-família, que se relaciona à análise da combinação das funções do trabalho e da família (Demerouti, Peeters \& van der Heijden, 2012).
Durante muito tempo, o lado negativo da interface trabalho-família foi o mais investigado, com destaque para o conflito trabalho-família, segundo o qual a participação em um domínio (ex., trabalho) dificulta a participação no outro (ex., família), uma vez que as demandas de um domínio não permitem que o indivíduo realize as demandas do outro domínio (Greenhaus \& Beutell, 1985). No entanto, o advento da Psicologia Positiva, nos anos 2000, fez com que construtos relacionados ao lado positivo dessa interface, como, por exemplo, o 
enriquecimento trabalho-família (a família pode beneficiar o trabalho e o trabalho pode beneficiar a família) também passassem a ocupar lugar de destaque na literatura dessa área (Greenhaus \& Powell, 2006).

$\mathrm{Na}$ tentativa de abordar tanto o lado negativo (conflito trabalho-família) quanto o lado positivo (enriquecimento trabalho-família) da interface trabalho-família, ten Brummelhuis e Bakker (2012) propuseram o Modelo de Recursos Trabalho-Família. Para os autores, o conflito e o enriquecimento trabalho-família constituem-se em dois processos em que as demandas contextuais (processo de conflito) e os recursos contextuais (processo de enriquecimento) de um domínio influenciam nos recursos pessoais dos indivíduos. Esses, por sua vez, influenciam nos resultados no outro domínio.

Embora o modelo em questão aborde tanto o lado positivo quanto o lado negativo da interface trabalho-família, o advento da Psicologia Positiva acabou levando a um aumento considerável das pesquisas sobre o lado positivo (enriquecimento trabalho-família), em detrimento das pesquisas sobre lado negativo (conflito trabalho-família) (Greenhaus \& Powel, 2006). Fundamentando-se em tais considerações, o presente trabalho prioriza o lado negativo do processo de interface trabalho-família. Nesse sentido, aborda as demandas do trabalho e as demandas da família, que se constituem em importantes preditoras do conflito do trabalho para a família e do conflito da família para o trabalho, respectivamente (Boyar, Carr, Mosley Jr. \& Carson, 2007). Tais demandas constituem-se, ainda, em importantes preditoras de recursos pessoais, bem como de diferentes resultados no trabalho e na família, tais como a satisfação, o bem-estar, o desempenho e o comprometimento no trabalho e na família (Brummelhuis \& Bakker, 2012).

As demandas estão associadas à percepção global do indivíduo acerca do seu nível de responsabilidade nas suas diferentes funções no trabalho e/ou na família. Dessa maneira, as demandas percebidas do trabalho relacionam-se à percepção da intensidade da responsabilidade que o indivíduo apresenta em seu trabalho. As demandas percebidas da família, por sua vez, referem-se à percepção quanto ao nível de responsabilidade que o indivíduo apresenta em sua família (Boyar et al., 2007).

Diferentes instrumentos têm sido elaborados com a finalidade de avaliar o conflito trabalho-família, como, por exemplo, as Escalas de Conflito TrabalhoFamília (Haslam, Filus, Morawska, Sanders \& Fletcher, 2015; Netemeyer, Boles \& Murrian, 1996) e a Escala de Interferência Trabalho-Família (Carlson \& Frone, 2003). Tais medidas, no entanto, não abordam os antecedentes do referido construto. Procurando preencher tal lacuna, Boyar, Carr, Mosley Jr. e Carson (2007) desenvolveram e reuniram evidências de validade, nos Estados Unidos, da Escala de Demandas Percebidas do Trabalho e da Família. A referida escala é composta de nove itens: cinco, para o fator demandas percebidas do trabalho, e quatro, para o fator demandas percebidas da família (Boyar et al., 2007).

No estudo original de desenvolvimento e validação do instrumento, participaram 997 trabalhadores estadunidenses (Boyar et a., 2007), divididos em três diferentes amostras: (a) 187 trabalhadores semiespecializados de fábricas do sul dos Estados Unidos; (b) 112 funcionários de presídios; e (c) 698 trabalhadores de uma universidade do sul dos Estados Unidos. Os resultados do estudo evidenciaram que a estrutura de dois fatores correlacionados foi a que apresentou os melhores índices de ajuste, e que os índices de consistência interna dos fatores, calculados pelo alfa de Cronbach, foram elevados nas três amostras. Foi observado, ainda, que as demandas percebidas do trabalho correlacionaram-se positivamente com a sobrecarga no trabalho, com o conflito de papéis no trabalho e com a ambiguidade de papéis no trabalho, enquanto as demandas percebidas da família correlacionaram-se positivamente com o conflito e com a ambiguidade de papéis na família (Boyar et al., 2007).

No que diz respeito a amostras brasileiras, todos os instrumentos de mensuração do lado negativo da interface trabalho-família, com evidências de validade para o contexto brasileiro, têm abordado tão somente o conflito trabalho-família, deixando de lado os possíveis antecedentes de tal fenômeno, como é o caso das demandas do trabalho e da família. Assim é que a consulta às bases de dados nacionais (SciELO e PePSIC), realizada em junho de 2018, com as palavras-chave "demandas do trabalho", "demandas da família" e "Escala de Demandas do Trabalho e da Família" apontou que a única escala do lado negativo da interface trabalho-família com evidências de validade obtidas no contexto brasileiro é a Escala de Conflito Trabalho-Família de Netemeyer, Boles e Murrian (1996), adaptada para amostras brasileiras por Aguiar e Bastos (2013).

No que tange aos estudos internacionais, a consulta às bases de dados internacionais (PsycInfo, SciELO e PePSIC), realizada em junho de 2018, com as palavras-chave "work demand", "family demand" e "Perceived Work and Family Demand Scale", revelou que o único estudo que apresenta evidências de validade da Escala de Demandas Percebidas do Trabalho e da Família é o de Boyar et al. (2007), já mencionado. Considerando, porém, que as demandas do trabalho e da família são importantes antecedentes dos processos de conflito trabalho-família (Boyar et al., 2007) e de diversos resultados no trabalho e na família (Brummelhuis \& Bakker, 2012), tornam-se necessários estudos brasileiros que contribuam para o preenchimento dessa lacuna. Ademais, a escala poderá auxiliar gestores organizacionais no desenvolvimento de intervenções para a melhoria da relação entre o trabalho e a família, ao explicitar os níveis de demandas percebidas do trabalho e da família que os indivíduos apresentam.

Fundamentando-se em tais considerações, o presente trabalho teve como objetivos: obter evidências iniciais de validade baseadas na estrutura interna e na invariância 
dos parâmetros dos itens do instrumento em termos de gênero, presença de filhos e ocupação ou não de cargo de chefia; verificar as relações com variáveis externas e identificar a validade discriminante da Escala de Demandas Percebidas do Trabalho e da Família, em uma amostra de trabalhadores brasileiros. Considerando-se que o instrumento original de Boyar et al. (2007) era composto de dois fatores, formulou-se a seguinte hipótese:

Hipótese 1: A versão brasileira da Escala de Demandas do Trabalho e da Família será composta de dois fatores: demandas do trabalho e demandas da família.

No que diz respeito à invariância dos parâmetros dos itens do instrumento, foram comparados três diferentes grupos, subdivididos quanto ao gênero, à presença de filhos e à ocupação ou não de cargos de chefia. Em relação ao gênero, pode-se afirmar que as mudanças sociais e a inserção das mulheres no mercado de trabalho mudaram a rotina tanto de homens quanto de mulheres. Elas, atualmente, têm menos tempo para se dedicar à família, o que fez com que os homens tenham passado a se dedicar mais ativamente à vida familiar. Entretanto, as mulheres continuam sendo as principais responsáveis pelas tarefas domésticas, apresentando, consequentemente, maior estresse no desempenho de seus papéis na família, em comparação aos homens (Neves \& Nascimento, 2017).

Quanto à presença de filhos, os estudos demonstram que as pessoas que têm filhos, principalmente as mulheres, apresentam maiores demandas na família, devido à sobrecarga de cuidado com ela (Neves \& Nascimento, 2017). No que tange à ocupação ou não de cargo de chefia, tem-se verificado que os indivíduos que ocupam cargos de chefia costumam perceber maiores demandas em seu trabalho, devido ao seu maior grau de reponsabilidade (Moro \& Amador, 2015). Em que pesem, porém, tais diferenças, seria desejável que os parâmetros dos itens da Escala de Demandas Percebidas do Trabalho e da Família fossem estáveis, isto é, que eles se mostrassem invariantes nesses diferentes grupos, o que garantiria o uso da escala neles (Damásio, 2013). Pautada nessas considerações, foi formulada a seguinte hipótese:

Hipótese 2: Os itens da Escala de Demandas Percebidas do Trabalho e da Família são invariantes em função do gênero, da presença de filhos e da ocupação ou não de cargos de chefia.

Para avaliar as relações do instrumento com variáveis externas, foram usados outros construtos a ele correlatos. No que tange às demandas percebidas do trabalho, foi utilizada a sobrecarga no trabalho, que diz respeito à percepção que o indivíduo tem sobre o excesso de tarefas que realiza, tanto em termos qualitativos quanto quantitativos, bem como sobre a indisponibilidade dos recursos necessários à execução de tais tarefas (Bowling \& Kirkendall, 2012). Seria de se esperar, assim, que os indivíduos que percebem que têm um alto grau de responsabilidade em seu trabalho sejam, também, aqueles que percebem que não dispõem do tempo necessário à conclusão de suas tarefas laborais. Nesse sentido, formulou-se a seguinte hipótese:

Hipótese 3: As demandas percebidas do trabalho apresentam uma correlação positiva de moderada a forte com a sobrecarga no trabalho.

No que diz respeito às demandas percebidas da família, foi utilizado o construto Ambiguidade de papéis na família, que se refere ao fato de os indivíduos não terem informações claras e precisas sobre suas tarefas, sua autoridade e seu próprio desempenho em sua família (Rizzo, House \& Lirtzman, 1970). Como tanto as demandas percebidas da família quanto a ambiguidade de papéis na família podem ser considerados estressores do contexto familiar, seria de se esperar que tais variáveis fossem positivamente correlacionadas. Fundamentando-se em tais considerações, formulou-se a seguinte hipótese:

Hipótese 4: As demandas percebidas da família apresentam correlação positiva de moderada a forte com a ambiguidade de papéis na família.

Em relação à validade discriminante, seria de se esperar que, apesar de as demandas do trabalho serem correlacionadas com a sobrecarga no trabalho, e as demandas da família apresentarem relações com a ambiguidade de papéis na família, tais construtos sejam minimamente diferentes, por não se tratar dos mesmos construtos. Em outras palavras, espera-se que os construtos referidos apresentem validade discriminante (Andrade \& Valentini, 2018). Nesse sentido, formularam-se as seguintes hipóteses:

Hipótese 5: As demandas percebidas do trabalho são minimamente diferentes da sobrecarga no trabalho, no sentido de a variância explicada de cada construto ser maior que os índices de correlações ao quadrado.

Hipótese 6: As demandas percebidas da família são minimamente diferentes da ambiguidade de papéis na família, no sentido de a variância explicada de cada construto ser maior que os índices de correlações ao quadrado.

Em síntese, com o objetivo de responder às hipóteses propostas, o presente trabalho testou as propriedades psicométricas da Escala de Demandas Percebidas do Trabalho e da Família, em trabalhadores brasileiros. Para tanto, obteve evidências iniciais de validade baseadas na estrutura interna e na invariância dos parâmetros dos itens do instrumento em termos de gênero, presença de filhos e ocupação ou não de cargo de chefia; verificou as relações dela com variáveis externas e identificou a validade discriminante do instrumento. A seguir, encontra-se o método utilizado para alcançar tais objetivos.

\section{Método}

\section{Participantes}

A amostra foi composta por 507 trabalhadores, de ambos os sexos, provenientes de 25 estados brasileiros, mais o Distrito Federal, com destaque para os estados de São Paulo (18,3\%), Rio de Janeiro (11,8\%) e Paraná 
$(10,8 \%)$. Do total de respondentes, a maioria era de mulheres $(56,8 \%)$. No que diz respeito ao estado civil, houve maior concentração de pessoas casadas (47,5\%), seguida de solteiras (41,6\%). Quanto à escolaridade, 27,8\% indicaram graduação completa, $27,2 \%$ mestrado completo e $20,9 \%$ pós-graduação completa. Em relação ao fato de ter ou não filhos, a maioria respondeu que $\operatorname{sim}(59,4 \%)$. No que tange ao fato de o indivíduo ocupar ou não cargo de chefia, a maior parte respondeu que não $(55,4 \%)$. A idade variou de 16 a 66 anos $(M=34,26 ; D P=10,02)$ e o tempo de serviço, de 1 a 48 anos $(M=10,12 ; D P=9,20)$. O critério de inclusão utilizado foi o de que os respondentes deveriam estar trabalhando e serem casados e/ou terem filhos, por se tratar de investigação no âmbito da interface trabalho-família. Dessa maneira, os participantes solteiros só foram incluídos na amostra quando tinham filhos.

\section{Instrumentos}

Escala de Demandas do Trabalho e da Família (Boyar et al., 2007). As demandas percebidas do trabalho e da família foram mensuradas pela Escala de Demandas do Trabalho e da Família, desenvolvida por Boyar et al. (2007), a qual se compõem de nove itens, divididos em dois fatores: demanda percebida do trabalho (cinco itens) e demanda percebida da família (quatro itens). Os itens foram respondidos por meio de escala do tipo Likert de cinco pontos $(1-$ discordo fortemente a 5 - concordo fortemente). Exemplo de item de demanda percebida do trabalho: "Meu trabalho exige toda a minha atenção". Exemplo de item de demanda percebida da família: "Sinto que tenho muitas demandas de família". O procedimento de tradução e retradução (back-translation) foi adotado para a tradução da escala (Borsa, Damásio, \& Bandeira, 2012). Tal procedimento consistiu em um professor fluente em inglês realizar uma tradução dos itens para o português; um professor de língua inglesa realizar tradução dessa versão novamente para o inglês; dois pesquisadores da área de psicometria realizarem uma revisão técnica e compararem essas duas versões, para verificar se houve equivalência semântica.

Escala de Sobrecarga no Trabalho (Spector \& Jex, 1998). A sobrecarga no trabalho foi avaliada pela Escala de Sobrecarga no Trabalho, desenvolvida por Spector e Jex (1998), que é unifatorial e se compõe de cinco itens, a serem respondidos em escalas tipo Likert de cinco pontos (1 - nunca a 5 - várias vezes por dia). Exemplo de item: "Com que frequência seu trabalho atual requer que você trabalhe muito rápido?". Considerando-se a inexistência de estudos anteriores sobre evidências de validade para o contexto brasileiro do referido instrumento, o presente trabalho investigou tais evidências, mediante a análise fatorial confirmatória (AFC), por meio da modelagem de equações estruturais, no software R ( R Core Team, 2017), no pacote Lavaan (Rossell, 2012). Os resultados da AFC demonstraram bons índices de ajuste (qui-quadrado=16,00(5), RMSEA $=0,07, \mathrm{CFI}=0,99 \mathrm{e}$
$\mathrm{TLI}=0,97)$ e confirmaram a estrutura unifatorial da escala, com a retenção de todos os itens. O índice de consistência interna, calculado pelo alfa de Cronbach, foi igual a 0,89 .

Escala de Ambiguidade de Papéis (Rizzo et al., 1970). Para a mensuração da ambiguidade de papéis na família, foi utilizada uma versão adaptada para a família da Escala de Ambiguidade de Papéis de Rizzo et al. (1970), que é unifatorial e composta de cinco itens, a serem respondidos em escalas tipo Likert de cinco pontos $(1-N a \tilde{o}$ se aplica de modo algum a 5 -Aplica-se totalmente). Exemplo de item: "Em minha família, não sei quais são as minhas responsabilidades". Devido à inexistência de estudos de evidências de validade da escala em amostras brasileiras, o presente trabalho obteve tais evidências iniciais, mediante a análise fatorial confirmatória (AFC), por meio da modelagem de equações estruturais, no software R ( $R$ Core Team, 2017), no pacote Lavaan (Rossell, 2012). Os resultados da AFC evidenciaram que a escala apresentou bons índices de ajuste $\left(\chi^{2}(5)=7,53\right.$, RMSEA $=0,03$, $\mathrm{CFI}=0,99$ e TLI $=0,99)$, além de confirmarem sua estrutura unifatorial, com a retenção de todos os itens. A escala obteve índice de consistência interna, calculado pelo alfa de Cronbach, igual a 0,80.

\section{Procedimentos}

A pesquisa foi submetida ao Comitê de Ética em Pesquisa da instituição dos autores e foi aprovada sob CAAE no 76517617.9.0000.5289. Os respondentes manifestaram sua concordância em participar da pesquisa mediante o preenchimento do Termo de Consentimento Livre e Esclarecido, no qual foi informado aos participantes o objetivo da pesquisa e foi garantida a preservação do sigilo das respostas individuais.

A coleta de dados foi realizada em versão on-line. Para tanto, foi criado um formulário no aplicativo Google Docs, com os instrumentos utilizados. O convite para participar da pesquisa foi enviado aos indivíduos por meio de mensagens no Facebook e pelo aplicativo Whatsapp, nos quais foi enviado o link para acessar o questionário. Antes de acessarem ao questionário, os participantes eram solicitados a preencher o Termo de Consentimento Livre e Esclarecido. Dessa forma, só era permitido o acesso ao questionário àqueles que concordavam em participar da pesquisa, de forma voluntária.

\section{Análise de dados}

Para verificar a estrutura da Escala de Demandas Percebidas do Trabalho e da Família (EDPTF), foi realizada a análise fatorial confirmatória, por meio da Modelagem de Equações Estruturais, no software R (R Core Team, 2017), no pacote Lavaan (Rossell, 2012). Para tanto, foi utilizado o estimador Weighted Least Square Mean and Variance Ajusted (WLSMV), declarando os itens como variáveis categórico-ordinais. Na referida análise, foram testados dois modelos distintos (um fator e 
dois fatores) para verificar qual o modelo que melhor se ajustava aos dados. Atendendo-se às recomendações de Brown (2006), foram analisados os seguintes índices de ajuste: qui-quadrado (testa a diferença entre a matriz empírica e a matriz do modelo teórico, sendo que quanto maior o valor do $\chi^{2}$ pior o ajustamento); Root-MeanSquare Error of Approximation (RMSEA - deve se situar abaixo de 0,08, aceitando-se valores até 0,10); TuckerLewis Index (TLI - são adequados modelos com valores acima de 0,95); Comparative Fit Index (CFI - são adequados valores acima de 0,95 ).

A consistência interna do instrumento foi calculada por meio do alfa de Cronbach. Para avaliar a invariância dos parâmetros dos itens entre os grupos separados por sexo, ter ou não filhos e ocupar ou não cargo de chefia, foi utilizada a modelagem de equações estruturais multigrupos (Damásio, 2013), no software R (R Core Team, 2017), no pacote sem Tools (Jorgensen, 2016). Nessa análise, foram testados modelos em que foram fixados o número de itens e fatores (invariância configural), as cargas fatoriais (invariância métrica) e os thresholds e scalars (invariância escalar). As diferenças entre os ajustes dos modelos foram avaliadas por meio da diferença do qui-quadrado $\left(\Delta \chi^{2}\right)$, do CFI $(\Delta \mathrm{CFI})$ e do RMSEA ( $\triangle$ RMSEA). Tais diferenças devem ser pequenas e praticamente desprezíveis. Dessa maneira, as diferenças de CFI e de RMSEA devem ser inferiores a 0,01 , de modo a indicar que as cargas fatoriais, os thresholds e scalars são invariantes entre os grupos analisados (Cheung \& Rensvold, 2002).

$\mathrm{Na}$ relação da Escala de Demandas Percebidas do Trabalho e da Família com variáveis externas, a normalidade dos escores foi inicialmente testada, por meio do Teste de Shapiro-Wilk. Como houve violação do pressuposto de normalidade, optou-se pelo uso do coeficiente de Spearman ( $\rho$ de Spearman), para o cálculo das correlações do instrumento com os escores globais das medidas a ele relacionadas, tendo-se considerado como fracas as correlações menores que 0,30 , como moderadas, as de 0,30 a 0,50 e, como fortes, as acima de 0,50 (Mukaka, 2012). A validade discriminante entre os fatores do instrumento e os construtos a eles correlacionados foi calculada por meio da Variância Média Extraída (VME). Nesse sentido, os valores da VME foram comparados com as correlações ao quadrado entre os construtos. Para afirmar que os construtos são minimamente diferentes entre si (validade discriminante), é necessário que os valores das VMEs sejam superiores aos índices de correlação ao quadrado (Valentini \& Damásio, 2016).

\section{Resultados}

$\mathrm{Na}$ análise da estrutura do instrumento, buscou-se testar dois diferentes modelos: o modelo de dois fatores correlacionados $\left(\chi^{2}(g l)=71,81(26)\right.$; CFI $=0,97$; TLI=0,96; RMSEA=0,06), conforme a estrutura do instrumento original de Boyar et al. (2007), e o modelo de um único fator $\left(\chi^{2}(g l)=975,49(27)\right.$; $C F I=0,85$; $\mathrm{TLI}=0,80$; RMSEA $=0,21)$, também testado por Boyar et al. (2007). Os resultados evidenciaram que o modelo de dois fatores correlacionados foi o que apresentou melhores índices de ajuste, em comparação ao modelo de um fator, confirmando a Hipótese 1. A Tabela 1 traz os fatores, os itens e suas cargas fatoriais padronizadas.

Tabela 1

Fatores, Itens e Cargas Fatoriais Padronizadas da Escala de Demandas Percebidas do Trabalho e da Família

\begin{tabular}{clc}
\hline Fator & \multicolumn{1}{c}{ Itens } & $\begin{array}{c}\text { Cargas Fatoriais } \\
\text { Padronizadas }\end{array}$ \\
\hline & Meu trabalho exige toda a minha atenção & 0,69 \\
& Sinto que tenho muitas demandas de trabalho & 0,85 \\
Demandas do Trabalho & Sinto que tenho muito o que fazer no trabalho & 0,82 \\
& Meu trabalho exige muito de mim & 0,88 \\
& Tenho recebido muito trabalho para fazer & 0,78 \\
& & 0,80 \\
Demandas da Família & Tenho que trabalhar duro em atividades relacionadas à minha família & 0,91 \\
& Minha família exige toda a minha atenção & 0,92 \\
& Sinto que tenho muitas demandas de família & 0,83 \\
\hline
\end{tabular}

A versão brasileira da Escala de Demandas Percebidas do Trabalho e da Família ficou, portanto, composta de dois fatores (demandas percebidas do trabalho e demandas percebidas da família), com cinco e quatro itens, respectivamente. Cumpre registrar que as cargas fatoriais variaram de 0,69 a $0,92(M=0,83)$, em uma demonstração de que os fatores explicam a maior parte da variância dos itens. Ademais, os índices de consistência interna dos fatores da escala, calculados pelo alfa de Cronbach, foram iguais a 0,90 (trabalho) e 0,92 (família). 
Considerando-se o modelo de estrutura de duas dimensões de primeira ordem encontrado, avaliou-se, por meio da análise fatorial multigrupo (AFCMG), a invariância dos parâmetros dos itens entre os grupos formados quanto ao gênero masculino e feminino, ter ou não filhos e ocupar ou não cargo de chefia. A análise dos dados obtidos (Tabela 2) revelou que as imposições de restrições trouxeram diferenças pequenas e praticamente desprezíveis nos indicadores analisados, ou seja, as diferenças de CFI e de RMSEA foram inferiores a 0,01. Tais resultados indicam que, para o modelo de dois fatores correlacionados de primeira ordem, as cargas fatoriais, os threshol$d s$ e scalars foram invariantes entre os grupos analisados (Cheung \& Rensvold, 2002), confirmando a Hipótese 2.

Tabela 2

Análise Fatorial Confirmatória Multigrupo (AFCMG)

\begin{tabular}{|c|c|c|c|c|c|c|}
\hline Gênero (homens $x$ mulheres) & $\chi^{2}(g l)$ & $\left(\Delta \chi^{2}\right)$ & CFI & $(\Delta \mathrm{CFI})$ & RMSEA & $(\triangle \mathrm{RMSEA})$ \\
\hline Configural & 139,94 & - & 0,973 & - & 0,082 & - \\
\hline Métrica & 153,77 & 13,82 & 0,971 & 0,002 & 0,080 & 0,002 \\
\hline Escalar & 163,98 & 10,21 & 0,970 & 0,001 & 0,077 & 0,003 \\
\hline Filhos (sim $x$ não) & $\chi^{2}(g l)$ & $\left(\Delta \chi^{2}\right)$ & CFI & $(\triangle \mathrm{CFI})$ & RMSEA & $(\triangle \mathrm{RMSEA})$ \\
\hline Configural & 145,91 & - & 0,971 & - & 0,084 & - \\
\hline Métrica & 172,51 & 26,60 & 0,965 & 0,006 & 0,087 & 0,003 \\
\hline Escalar & 208,03 & 35,52 & 0,956 & 0,009 & 0,092 & 0,005 \\
\hline Cargo de chefia (sim $x$ não) & $\chi^{2}(g l)$ & $\left(\Delta \chi^{2}\right)$ & CFI & $(\Delta \mathrm{CFI})$ & RMSEA & $(\triangle \mathrm{RMSEA})$ \\
\hline Configural & 143,66 & - & 0,972 & - & 0,083 & - \\
\hline Métrica & 153,51 & 9,84 & 0,971 & 0,001 & 0,079 & 0,004 \\
\hline Escalar & 164,01 & 10,51 & 0,970 & 0,001 & 0,077 & 0,003 \\
\hline
\end{tabular}

Notas. $\chi^{2}=$ qui-quadrado; $\Delta \chi^{2}=$ diferença de qui-quadrado; CFI=Comparative Fix Index; $\Delta$ CFI=diferença do CFI; RMSEA=Root Mean Square Error of Approximation; $\triangle$ RMSEA=diferença do RMSEA. Configural=estrutura fatorial fixa entre os grupos; Métrica=estrutura fatorial e cargas fatoriais fixas entre os grupos; Escalar=estrutura fatorial, cargas fatoriais, thresholds e scalars fixos entre os grupos. $n$ homens $=219 ; n$ mulheres=288; $n$ tem filhos $=206 ; n$ não tem filhos=301; $n$ ocupa cargo de chefia=226; $n$ não ocupa cargo de chefia=281

Na verificação da relação do instrumento com variáveis externas, os escores obtidos em cada um dos instrumentos de coleta de dados foram computados, a partir da média dos escores de seus respetivos itens e as médias e correlações entre essas escalas foram calculadas. Os dados obtidos no cálculo dessas correlações evidenciaram que a correlação entre as demandas percebidas do trabalho e a sobrecarga no trabalho foi forte e positiva $(\rho=0,75$; $p<0,01$ ), o que confirma a Hipótese 3; e que a correlação entre as demandas percebidas da família e a ambiguidade de papéis foi fraca e negativa $(\rho=-0,11 ; p<0,05)$, o que impediu a confirmação da Hipótese 4 (Tabela 3 ).

Tabela 3

Médias, Desuios Padrão e Correlações de Spearman entre as Demandas Percebidas do Trabalho e da Família com Variáveis Externas

\begin{tabular}{|c|c|c|c|c|c|}
\hline Variáveis & Média & $D P$ & 1 & 2 & 3 \\
\hline 1. Demanda percebida do trabalho & 3,86 & 0,96 & - & - & - \\
\hline 2. Demanda percebida da família & 3,23 & 1,20 & $0,38^{* *}$ & - & - \\
\hline 3. Sobrecarga no trabalho & 3,60 & 0,99 & $0,75^{* *}$ & $0,40^{* *}$ & - \\
\hline 4. Ambiguidade de papéis na família & 3,77 & 0,97 & $0,19^{* *}$ & $-0,11^{*}$ & $0,19^{* *}$ \\
\hline
\end{tabular}
Nota. ${ }^{*} p<0,05 ;{ }^{* *} p<0,01$

Para a avaliação da validade discriminante da Escala de Demandas Percebidas do Trabalho e da Família, os valores das VMEs foram comparados com os índices de correlação ao quadrado, sendo que o valor da VME do modelo composto pelo fator demandas percebidas do trabalho e pela sobrecarga no trabalho $(0,63)$ foi superior ao índice de correlação ao quadrado entre os construtos $\left(\rho^{2}=0,56\right)$, confirmando a Hipótese 5 . Além disso, o valor da VME do modelo composto pelo fator demandas percebidas da família e pela ambiguidade de papéis na 
família $(0,65)$ foi superior ao índice de correlação ao quadrado entre as variáveis $\left(\rho^{2}=0,01\right)$, o que confirmou a Hipótese 6.

\section{Discussão}

O presente trabalho teve como finalidade investigar as evidências de validade de estrutura interna e de consistência interna da Escala de Demandas Percebidas do Trabalho e da Família, bem como avaliar a invariância dos parâmetros dos itens do instrumento em termos de gênero, ter ou não filhos e ocupar ou não cargo de chefia. Além disso, procurou verificar a relação do instrumento com variáveis externas e sua validade discriminante.

Os dados obtidos na análise fatorial confirmatória revelaram que o modelo de dois fatores correlacionados foi o que apresentou os melhores índices de ajuste, confirmando a Hipótese 1. Tais resultados mostram-se consistentes com o estudo de construção e validação do instrumento original (Boyar et al., 2007), no qual também foi obtida uma estrutura interna de dois fatores correlacionados. Assim, a versão final brasileira da Escala de Demandas Percebidas do Trabalho e da Família manteve a estrutura de dois fatores (com cinco e quatro itens, respectivamente) do modelo original de Boyar et al. (2007). Logo, a presente pesquisa adiciona evidências transculturais à conclusão de que o modelo no qual as demandas do trabalho e da família estruturam-se como fatores distintos é o mais adequado à descrição do instrumento.

Os indicadores de precisão do alfa de Cronbach apresentaram valores altos e superiores aos encontrados por Boyar et al. (2007). Tais resultados indicam que, no presente trabalho, a correlação entre os itens foi mais elevada que no estudo citado. Essa divergência pode se dever a diferenças culturais entre as amostras. Em outras palavras, é possível que os brasileiros apresentem maior tendência a responder, em uma mesma direção, a instrumentos que apresentem quantidade reduzida de itens. Tal fato pode ter elevado o nível de consistência interna da escala na amostra brasileira e merece ser explorado com maior profundidade em investigações futuras. De todo modo, os resultados indicam que os escores dos dois fatores podem ser estimados com alta precisão, mesmo com a quantidade reduzida de itens, em amostras de nacionalidades distintas. Foi possível, assim, constatar que os escores do instrumento são minimamente precisos, isto é, com poucos erros de medição devido à falta de consistência interna (Cortina, 1993).

No que se refere à invariância dos parâmetros dos itens do instrumento, buscou-se avaliar se a estrutura fatorial encontrada poderia ser considerada invariante para homens e mulheres, indivíduos com ou sem filhos e ocupantes ou não de cargos de chefia. Conforme esperado, os resultados demonstraram que os escores de demandas percebidas do trabalho e da família obtidos por meio da escala são invariantes para os diferentes grupos analisados. Tais resultados corroboraram a Hipótese 2, segundo a qual o instrumento pode ser utilizado indistintamente entre esses grupos, sem vieses de resposta (Damásio, 2013).

No que diz respeito à relação da escala com variáveis externas, observou-se que as demandas percebidas do trabalho apresentaram correlação positiva forte com a sobrecarga no trabalho. Tal achado confirma a Hipótese 3 e mostra-se consistente com os relatos de Boyar et al. (2007), que também encontraram correlações positivas fortes entre tais construtos. Apesar de as demandas percebidas do trabalho referirem-se a exigências mais gerais associadas ao alto grau de responsabilidade na execução de tarefas laborais (Boyar et al., 2007), a sobrecarga no trabalho leva as pessoas a se depararem com obrigações que exigem que elas façam mais do que têm condições de fazer no tempo disponível (Bowling \& Kirkendall, 2012). Logo, ambas fazem exigências aos trabalhadores, o que explicaria a relação positiva entre elas.

O presente estudo evidenciou, ainda, que as demandas percebidas da família apresentaram correlação negativa fraca com a ambiguidade de papéis na família, o que impediu a confirmação da Hipótese 4. Tal resultado é contrário aos achados de Boyar et al. (2007), que encontraram correlações positivas fracas entre tais variáveis. Uma possível explicação para tal resultado é a de que quando o indivíduo apresenta um alto nível de responsabilidade em sua família (Boyar et al., 2007), ele provavelmente tende a se conscientizar melhor acerca dos papéis a desempenhar nesse contexto e, consequentemente, teria maior clareza sobre as tarefas a cumprir.

Os achados da pesquisa demonstraram, também, que o valor da VME do modelo composto pelo fator demandas percebidas do trabalho e pela sobrecarga no trabalho foi superior ao índice de correlação ao quadrado entre os construtos, além de que o valor da VME do modelo composto pelo fator demandas percebidas da família e pela ambiguidade de papéis na família também foi superior ao índice de correlação ao quadrado entre as variáveis, o que permitiu a confirmação das Hipóteses 5 e 6. Tais resultados demonstram, portanto, que os fatores demandas percebidas do trabalho e demandas percebidas da família são minimamente diferentes da sobrecarga no trabalho e da ambiguidade de papéis na família, respectivamente (Valentini \& Damásio, 2016).

Em síntese, a Escala de Demandas Percebidas do Trabalho e da Família apresentou evidências adequadas de validade baseada na estrutura interna, na invariância dos parâmetros dos itens e nas relações com outros construtos e discriminante. Cumpre, porém, registrar as limitações do estudo. A primeira delas refere-se ao fato de que o instrumento utilizado foi de autorrelato e nem sempre o que é relatado pelos participantes condiz com a realidade. Outra limitação é que a amostra, em sua totalidade, possuía, pelo menos, o ensino médio, sendo que maioria 
tinha pelo menos graduação completa. Desse modo, não é possível generalizar os resultados para amostras de indivíduos sem ensino médio completo. Além disso, a coleta de dados foi realizada apenas on-line, sem a presença dos pesquisadores, o que pode ter gerado falta de comprometimento e respostas menos confiáveis. Merece destaque, ainda, o fato de o estudo ter se focado apenas no lado negativo da interface trabalho-família, deixando, assim, de abordar importantes variáveis que impactam o lado positivo dessa interface, como, por exemplo, o apoio e a motivação. Por fim, o estudo foi de natureza transversal, o que impossibilita inferir uma relação de causalidade entre as variáveis.

No que tange a uma agenda futura de pesquisa, sugere-se a realização de estudos destinados a buscar evidências adicionais sobre a validade preditiva da escala, mediante sua correlação com comportamentos negativos emitidos no contexto do trabalho (por exemplo, absenteísmo) e da família (por exemplo, atraso para chegar em casa). Investigações adicionais poderiam, ainda, aprofundar a rede nomológica das demandas percebidas do trabalho e da família, principalmente no que tange a seus impactos em resultados do trabalho e da família (como, por exemplo, a satisfação, o bem-estar, o desempenho e o comprometimento). Essas pesquisas deveriam ser, preferencialmente, de natureza longitudinal, com o objetivo de obter maior compreensão acerca das relações entre essas variáveis. Estudos futuros com a escala em grupos específicos, como, por exemplo, o de trabalhadores flexíveis (home office) e o de trabalhadores que estão vivenciando as novas configurações familiares, também poderiam ser realizados.

De todo o modo, os resultados obtidos permitem a conclusão de que a versão brasileira da Escala de Demandas Percebidas do Trabalho e da Família mostrou-se apropriada à mensuração da percepção do grau de responsabilidade que o indivíduo apresenta em seu trabalho e em sua família, o que recomenda seu uso futuro em pesquisas com foco na interface trabalho-família. Além disso, o instrumento poderá auxiliar em intervenções cujo foco seja a melhoria da relação entre o trabalho e a família, ao fornecer um diagnóstico dos níveis de demandas percebidas do trabalho e da família entre os trabalhadores.

\section{Referências}

Aguiar, C. V. N., \& Bastos, A. V. B. (2013). Tradução, adaptação e evidências de validade para a medida de conflito trabalho-família. Avaliação Psicológica, 12(2), 203-213. Recuperado de http://pepsic.bvsalud.org/scielo.php?script=sci_arttext\&pid=S1677-047120130002 00011\&lng $=$ pt\&tlng $=$ pt

Allen, T. D., Cho, E., \& Meier, L. L. (2014). Work-family boundary dynamics. Annual Review of Organizational Psychology and Organizational Behavior, 1(1), 99-121. doi: 10.1146/annurev-orgpsych-0314130091330

Andrade, J. M., \& Valentini, F. (2018). Diretrizes para a construção de testes psicológicos: A resolução CFP no 009/2018 em destaque. Psicologia: Ciência e Profissão, 38 (núm. esp.), 28-39. doi: 10.1590/1982-3703000208890

Borsa, J. C., Damásio, B. F., \& Bandeira, D. R. (2012). Adaptação e validação de instrumentos psicológicos entre culturas: Algumas considerações. Paidéia, 22(53), 423-432. doi: 10.1590/S0103-863X2012000300014

Boyar, S. L., Carr, J. C., Mosley Jr., D. C., \& Carson, C. M. (2007). The development and validation of scores on Perceived Work and Family Demand Scales. Educational and Psychological Measurement, 67(1), 100-115. doi: 10.1177/0013164406288173

Bowling, N. A., \& Kirkendall, C. (2012). Workload: A review of potential causes, consequences, and interventions. Em J. Houdmont, S. Leka, \& R. Sinclair (Eds.), Contemporary occupational health psychology: Global perspectives on research and practice (Vol. 2, pp. 221-238). Chichester: Wiley-Blackwell.

Brown, T. A. (2006). Confirmatory factor analysis for applied research. New York: Guilford.

Carlson, D. S., \& Frone, M. R. (2003). Relation of behavioral and psychological involvement to a new four-factor conceptualization on work-family interference. Journal of Business Psychology, 17(4), 515-535. doi: 10.1023/A:1023404302295

Cortina J. (1993) What is coefficient alpha: An examination of theory and applications. Journal Applied of Psychology, 78(1), 98-104. doi:10.1037/0021-9010.78.1.98

Damásio, B. F. (2013). Contribuições da análise fatorial confirmatória multigrupo (AFCMG) na avaliação de invariância de instrumentos psicométricos. Psico-USF, 18(2), 211-220. doi: 10.1590/S1413-82712013000200005

Demerouti, E., Peeters, M. C. W., \& van der Heijden, B. I. J. M. (2012). Workfamily interface from a life and career stage perspective: The role of demands and resources. International Journal of Psychology, 47(4), 241-258. doi: 10.1080/00207594.2012.699055

Greenhaus, J., \& Powell, G. (2006). When work and family are allies: A theory of work-family enrichment. Academy of Management Review, 31(1), 72-92. doi: 10.5465/AMR.2006.19379625

Haslam, D., Filus, A., Morawska, A., Sanders, M. R., \& Fletcher, R. (2015). The Work-Family Conflict Scale (WAFSC): Development and initial validation of a self-report measure of a work-family conflict for use with parents. Child Psychiatry and Human Development, 46(3), 346-357. doi: 10.1007/s10578-014-0476-0

Jorgensen, T. D. (2016). semTools: Useful tools for structural equation modeling. Recuperado de https://CRAN.R-project.org/package=semTools

Moro, C. V. M., \& Amador, F. S. (2015). O trabalho da gestão: Notas sobre poder e subjetividade. Revista Psicologia Organizações e Trabalho, 15(2), 201-211. doi: 10.17652/rpot/2015.2.511

Mukaka M. M. (2012). Statistics corner: A guide to appropriate use of correlation coefficient in medical research. Malawi Medical Journal, 24(3), 69-71. Recuperado de https:/www.ncbi.nlm.nih.gov/pmc/articles/PMC3576830/ 
Netemeyer, R. G., McMurrian, R., \& Boles, J. S. (1996). Development and validation of work-family conflict and family-work conflict scales. Journal of Applied Psychology, 81(4), 400-410. doi: 10.1037/0021-9010.81.4.400

Neves, D. R., \& Nascimento, R. P. (2017). A divisão entre trabalho, família e organizações para casais de dupla jornada (two-job couples): Notas para um debate sobre o caso brasileiro. Economia \& Gestão, 17(48), 157-173. doi: 10.5752/P.1984-6606.2017v17n48p157-173

R Core Team. (2017). R: A language and environment for statistical computing. R Foundation for Statitiscal Computing, Vienna, Austria. Recuperado de http://www.R-project.org/

Rizzo, J. R., House, R. J., \& Lirtzman, S. I. (1970). Role conflict and ambiguity in complex organizations. Administrative Science Quarterly, 15(1), 150-163. doi: 10.2307/23911486

Rossell, Y. (2012). Lavaan: An R package for structural equation modeling. Journal of Statistical Software, 48(2), 1-36. Recuperado de http:// www.jstatsoft.org/ v48/i02/paper

Spector, P. E., \& Jex, S. M. (1998). Development of four self-report measures of job stressors and strain: Interpersonal Conflict at Work Scale, Organizational Constraints Scale, Quantitative Workload Inventory, and Physical Symptoms Inventory. Journal of Occupational Health Psychology, 3(4), 356-367. doi: 10.1037/1076-8998.3.4.356

ten Brummelhuis, L. L., \& Bakker, A. B. (2012). A resource perspective on the work-home interface: The Work-Home Resources Model. The American Psychologist, 67(7), 545-556. doi: 10.1037/a0027974

Valentini, F., \& Damásio, B. F. (2016). Variância média extraída e confiabilidade composta: Indicadores de precisão. Psicologia: Teoria e Pesquisa, 32(2), 1-7. doi: 10.1590/0102-3772e322225

\section{Sobre as autoras}

Larissa Maria David Gabardo-Martins é psicóloga e doutoranda em Psicologia pela Universidade Salgado de Oliveira, Niterói, RJ. Principais temas de atuação: psicologia positiva, comportamento organizacional positivo, bem-estar, florescimento e interface trabalho-família.

Maria Cristina Ferreira é psicóloga, doutora em Psicologia pela Fundação Getúlio Vargas, professora titular e coordenadora do Programa de Pós-Graduação em Psicologia, da Universidade Salgado de Oliveira, Niterói, RJ. Principais temas de estudo: psicologia positiva, qualidade de vida, bem-estar, engajamento, comportamento organizacional positivo. 